\title{
Epistemologías del Sur y las Economías de Ellas. La Cooperativa "Capuchinhas" de la Sierra de Montemuro"
}

\author{
Teresa Cunha ${ }^{2}$, Vanessa Sousa ${ }^{3}$
}

Recibido: 7 de febrero de 2019/Aceptado: 27 de abril de 2019

\begin{abstract}
Resumen. La cooperativa "Capuchinhas" es una iniciativa de mujeres de Serra do Montemuro, una de las regiones más aisladas y empobrecidas del centro de Portugal. Desde su creación en la década de 1980, esta cooperativa ha sido ideada como forma de resistencia al empobrecimiento y al aislamiento geográfico. Por otro lado, se ha convertido en una alternativa local para la creación de empleo y la emancipación de las mujeres. Esta cooperativa presta especial atención a los problemas concretos de su región y su población, y busca afrontar los desequilibrios territoriales y la creciente mercantilización de todos los ámbitos de la vida. Nuestro trabajo tiene dos objetivos principales. El primero es la expansión teórica y analítica del concepto de economía feminista. En segundo lugar, a través de una etnografía densa, presentamos la complejidad del establecimiento y la vida de la cooperativa, dando centralidad a las propias palabras y narrativas de las protagonistas que piensan en sí mismas y en el mundo en el que viven y trabajan.
\end{abstract}

Palabras clave: Economía feminista; cooperativa de mujeres; trabajo productivo; Capuchinhas; epistemologías del Sur.

\section{[en] Epistemologies of the South and Her Economy. The 'Capuchinhas' cooperative of the Serra de Montemuro}

\begin{abstract}
The 'Capuchinhas' cooperative is an initiative of women from Serra do Montemuro, one of the most isolated and impoverished regions of the interior of Portugal. Since its beginning in the 1980 s, this cooperative was thought of as a form of resistance to impoverishment and geographical segregation. In addition, it constituted a local alternative to unemployment and women's emancipation. This co-operative pays special attention to the specific problems of its region and its population and seeks to tackle territorial imbalances and the increasing commodification of all spheres of life. Our work has two main objectives. The first is the theoretical and analytical extension of the concept of feminist economy. Secondly, through a dense ethnography we present the complexity of the creation and life of the cooperative, placing the words and narratives of the protagonists, who think about themselves and the world where they act, at the center of the analysis.
\end{abstract}

Keywords: Feminist economics; cooperative of women; productive work; Capuchinhas; epistemologies of the South.

Sumario. 1. Introducción. 2. Las epistemologías del Sur y las economías de Ellas. 3. La cooperativa "Capuchinhas": donde lo personal es político y lo político es personal. 3.1. Transformar conservando: el contexto y el origen de la cooperativa. 3.2. La imaginación polifónica de la emancipación de la mujer. 3.2.1 Objetos que son más que objetos y otra economía del deseo. 3.2.2. Quiero todo y quiero

\footnotetext{
Traducción al castellano por Fernando Barbosa Rodrigues (UCM)

Centro de Estudos Sociais da Universidade de Coimbra, Portugal. teresacunha@ces.uc.pt

Associação Montanhas de Saberes. vanessa.alcantara.sousa@gmail.com
} 
ser yo quien diga qué es, todo, para mí. 3.3. Recursos locales, conocimiento y tecnologías antiguas e innovación para una economía de abundancia. 3.3.1 Obligando al mercado a estar al servicio de la transformación de la vida. 3.3.2. El tiempo no es dinero ni el dinero puede ser el tiempo. 4. Notas finales. 5. Bibliografía.

Cómo citar: Cunha, T.; Sousa, V. (2019). Epistemologías del Sur y las Economías de Ellas. La Cooperativa "Capuchinhas" de la Sierra de Montemuro, en Revista de Antropología Social 28(2), 323-344.

\section{Introducción}

Las "Capuchinhas" es una cooperativa diseñada y dirigida por mujeres del pueblo de Campo Benfeito ${ }^{4}$ en la Sierra de Montemuro, una de las regiones más aisladas y empobrecidas del centro de Portugal. Sus 31 años de vida ${ }^{5}$ muestran que es una iniciativa socioeconómica sostenible arraigada en su territorio. Con el tiempo, ha dado pruebas de una notable capacidad para producir innovación basada en un diálogo cercano entre el conocimiento ancestral, los recursos locales y las estrategias de emancipación de las mujeres.

El establecimiento de las "Capuchinhas" fue el resultado de la participación y la articulación colaborativa de varias organizaciones sociales y personas que, a mediados de la década de 1980, se sintieron comprometidas con la democratización de la sociedad portuguesa para romper el viejo orden heredado del fascismo que el país vivió durante 48 años $^{6}$. En aquel contexto, quedó claro que era necesario intervenir en los territorios a varios niveles. Por un lado, el objetivo era impulsar los tejidos productivos capaces de sostener las economías locales y el asentamiento de poblaciones marcadas por las sucesivas oleadas de emigración, como una forma de escapar del empobrecimiento al que habían estado sometidas durante décadas. Por otro lado, el objetivo era crear e implementar herramientas de aprendizaje que permitieran a las personas llevar a cabo sus propios proyectos de vida y desarrollo económico de manera autónoma. Entre todos los grupos sociales involucrados, se prestó especial atención a las mujeres, su participación activa y el reconocimiento de su trabajo y papel en una sociedad que quería convertirse en una comunidad moderna y avanzada, que respetara el principio constitucional de igualdad entre mujeres y hombres. Es en esta atmósfera de democratización y transformación que podemos entender mejor, no solo el surgimiento de la cooperativa de las "Capuchinhas" sino su importancia. En Portugal, especialmente en la región donde se ubica, la cordillera de Montemuro, esta cooperativa sigue siendo una de las experiencias concretas más largas e interesantes de una economía basada en el pluriverso del trabajo de las mujeres, capaz de crear riqueza y mejorar el territorio en sus diversas formas. No es cosa baladí el nombre que han elegido para identificarse: "Capuchinhas". Se refiere tanto al tejido de lana, el burel ${ }^{7}$, con el que se confeccionan las capuchas, que fueron y siguen siendo la ropa de abrigo para el invierno de la gente de la región, como a

\footnotetext{
Campo Benfeito pertenece a la parroquia de Gosende, Consejo de Castro Daire, distrito de Viseu, Portugal.

Podrá escucharse un testimonio corto sobre las "Capuchinhas" aquí:

https://www.imagensdemarca.pt/artigo/capuchinhas-4-mulheres-que-dao-uma-nova-vida-ao-burel/

6 Portugal vivió una dictadura militar instaurada el 28 de mayo de 1926 con carácter de régimen fascista hasta el 25 de Abril de 1974, cuando se dio la revolución democrática, también llamada la "revolução dos cravos" (revolución de los claveles).

7 Un burel es un tejido de lana prensado tradicionalmente en un dispositivo llamado pisotón. Hoy en día el burel ya se obtiene por procesos mecánicos industriales.
} 
muchos de los trabajos y tareas que hacen las mujeres: desde el pastoreo de ovejas, el hilado de la lana, el corte del burel, la fabricación y decoración de estos objetos tan particulares y necesarios para enfrentar el duro clima de la sierra de Montemuro.

Con el propósito de poner en diálogo una hermenéutica feminista (Cunha, 2017) de las epistemologías del Sur, de Santos $(2014,2018)$, y las prácticas socioeconómicas colectivas de las "Capuchinhas", nuestro trabajo se estructura en dos partes principales. En la primera parte, aunque de forma breve, discutimos teóricamente dos ideas relacionadas con la ampliación del concepto de economía feminista. La primera es la persistencia de las condiciones de subordinación e invisibilidad de una parte de la humanidad, que son las mujeres; la segunda es la invención de la dicotomía entre el trabajo productivo y reproductivo y cómo su deconstrucción arroja un nuevo desafío para los feminismos y las economías feministas.

En la segunda parte, más larga y detallada, presentamos una etnografía de las "Capuchinhas" para llevar a la discusión la complejidad de la creación y la vida de la cooperativa. Buscamos entender cómo, a lo largo de su existencia, ha tratado de responder a los desafíos planteados por la mercantilización progresiva de la vida y la economía del país, la sostenibilidad ambiental y económica, y el lugar del conocimiento y el trabajo de las mujeres para resistir y crear alternativas concretas y viables. En esta segunda parte, la coautoría es de cinco mujeres de la comunidad de Campo Benfeito que, con nosotras, narran, piensan y señalan el camino para el presente y el futuro.

Nuestra metodología etnográfica está traspasada por tres dimensiones, todas las cuales constituyen un proceso que es reflexivo y crítico. La primera es que 5 de las 6 autoras de la segunda parte del texto son parte de la cooperativa. Por tanto, es un proceso de autorreflexión y escritura colaborativa sobre sus vidas, la historia de la cooperativa y los momentos considerados cruciales y que definen su identidad y sus estrategias económicas. La segunda dimensión es el diálogo que ha existido durante muchos años, más de dos décadas, entre las autoras y el conocimiento y la interacción mutua en varias etapas de la vida de la cooperativa. Esto significa que recurrimos a un ejercicio prolongado de observación y participación en eventos formales e informales de la vida y actividades de la cooperativa en el pueblo y más allá. La tercera dimensión de la etnografía ha contado con entrevistas a varias de las protagonistas del proceso, el análisis de documentos de periódicos y literatura gris que nos ayudó a completar algunos borrados de la memoria oral y a recuperar una información más técnica que fueron consideradas irrelevantes por las subjetividades resistentes (Lugones, 2010: 746) de la cooperativa "Capuchinhas".

\section{Las epistemologías del Sur y las economías de Ellas}

Dado que nuestro punto de partida es el de las epistemologías del Sur desarrolladas por Boaventura de Sousa Santos $(2014,2018)$, queremos ampliarlo desde una hermenéutica feminista (Cunha, 2017). Esto significa, entre otras cosas, hacer que algunas de sus preguntas teóricas sean más visibles con respecto a la subalternidad construida de las mujeres y la dicotomía entre el trabajo productivo y reproductivo.

Compartimos la idea de que el Sur, al que se refiere este campo teórico, no es un lugar geográfico. Es una metáfora de los sufrimientos y el conocimiento forjados en las luchas contra todas las formas de opresión y dominación. Son la afirmación de 
que este conocimiento es válido para pensar y contar las experiencias de resistencia y la existencia de alternativas y, además, es necesario que la mayoría de las personas en el mundo lo representen como propio. Por tanto, se trata de discernir, identificar y valorar todas las energías cognitivas existentes que permiten y nutren la vida en todas sus facetas, transformación, liberación y emancipación. Tiene sentido, entonces, afirmar que la justicia social siempre implica justicia cognitiva (Santos, 2014: 133).

Sin embargo, y sabiendo que, a lo largo de la historia, las mujeres ${ }^{8}$ son el grupo humano más sometido por la mayoría de los sistemas y capas de opresión (Cunha, 2014), este carácter transversal, estructural y plural de sus experiencias de victimización, pero también de emancipación, debe ser completamente visible y crucial para las epistemologías del Sur. Por tanto, afirmamos que no hay justicia social y cognitiva sin justicia sexual. Es decir, no hay justicia social y cognitiva hasta que todas las mujeres y otras identidades que se representan a sí mismas como femeninas, así como sus prácticas, conocimiento y trabajo, estén libres de cualquier forma de discriminación, violencia o subalternización. Así pues, cuestionamos cualquier epistemología, feminista o de otro tipo, que no resalte las diversas estructuras de poder que, por un lado, homogeneizan y amalgaman la diversidad potencialmente infinita del conocimiento y la experiencia de las mujeres; y, por otro lado, no cuestionan las relaciones de poder que, de una forma u otra, en la producción y en el trabajo continúan socavando sus funciones, actividades, formas de vida y de nombrar el mundo. Argumentamos que una hermenéutica feminista de epistemologías del Sur nos llama a deconstruir cualquier aparato androcéntrico que esté, explícita o implícitamente, presente en nuestros análisis y teorizaciones, por muy críticos que presuman ser.

Por otro lado, una hermenéutica feminista de las epistemologías del Sur permite distinguir más claramente la racionalidad sexista ${ }^{9}$ que está presente en el pensamiento económico capitalista de la matriz occidental moderna. Las economistas feministas destacan su carácter androcéntrico que se revela de diversas maneras a nivel epistemológico, político y metodológico (Federici, 2013; Orozco y Calderón, 2018: 6). Una lectura de una economía feminista destaca los siguientes elementos de diferenciación que deben tenerse en cuenta en esta discusión. La primera es la afirmación de que la economía es mucho más que mercados y relaciones monetarias. Como veremos a continuación, la economía se refiere a toda la sociabilidad que sustenta y garantiza la producción de vida en todas sus formas (Carrasco, 2003; Osório, 2014; Echániz, 2018; Mies, 2019). Por tanto, todo el trabajo de las mujeres, que se ha hecho invisible y se considera que está fuera de la economía, es

8 Como no es el objetivo de nuestro texto, no desarrollamos la discusión sobre el potencial colonial y heteronormativo del concepto de "mujer", considerado como universal. Este tema ha sido abordado en la literatura por autoras como Ifi Amadiume (1998), Catarina Martins, (2016), Teresa Cunha (2014, 2015), Chandra Talpade Mohanty (1991), Linda Carty y Chandra Talpade Mohanty (2014), María Lugones (2010), Karina Bidaseca y Vanesa Laba (2011), Sílvia Cusicanquí (2010), Judith Butler (2004), Oyewùmí Oyèrónké (2005), entre muchas otras. Sin embargo, observamos aquí, no solo el debate, sino también nuestra atención al mismo y nuestro acuerdo con las críticas llevadas a cabo por estas y otras feministas.

9 Nada como los temas de acoso sexual que han sido discutidos y hechos visibles por movimientos como "Yo también" - Me Too - , que muestra este carácter androcéntrico constitutivo en nuestras sociedades. De hecho, lo que "Yo también" ha demostrado es que la transversalidad y la escala del acoso sexual contra las mujeres es un problema estructural en el que están involucradas mujeres y hombres, y que cualquier resolución seria del problema depende de todos. Por el contrario, las reacciones masculinas, en lugar de plantearse como parte del problema, dan lugar a soluciones para una mayor discriminación y segregación de las mujeres. Véase como ejemplo las noticias sobre las estrategias de los hombres en Wall Street descritas aquí.: https://www.publico. pt/2018/12/03/culturaipsilon/noticia/wall-street-homens-ja-nao-querem-viajar-lado-mulheres-1853345. 
una parte integral del pensamiento económico feminista. En segundo lugar, trata la división sexual del trabajo como motivada políticamente y construida socialmente (Orozco, 2014; Cunha, 2015, 2017; Orozco y Calderón, 2018). Esto tiene como principales consecuencias la desnaturalización y el cuestionamiento de los fundamentos de una economía en la que el hombre es el cabeza de familia (Mies, 2019: 396) y la medida de todas las cosas. Este desafío al sexismo de una economía que no se cuestiona sobre los fundamentos de las desigualdades sociales que genera y alimenta está bien expresado en los títulos de algunos trabajos de economistas feministas como Katrine Marçal (2016), Rachel Waterhouse y Carin Vijfhuizen (2001) respectivamente: Who cooked Adam Smith's Dinner? y Estratégias das mulheres, proveitos dos homens.

En tercer lugar, destacamos algunas diferencias metodológicas. Para una economía feminista, en lugar de utilizar las matemáticas y la lógica hipotético-deductiva para describir, comprender y analizar fenómenos económicos, lo importante es comprometerse a superar las desigualdades entre mujeres y hombres en el ámbito social, en general, y económico, en particular. Esto requiere incorporar todos los trabajos, aquellos que son remunerados y no remunerados. Asumir que todo conocimiento es político y contextual quiere decir que ser hombre o mujer, no solo importa, sino que debe ser central en las metodologías de producción de conocimiento económico (Orozco y Calderón, 2018: 7). Y finalmente, considera como su propósito la autonomía de los cuerpos y las vidas de las personas que se representan a sí mismas como femeninas y su capacidad para producir y mantener la vida a través de su trabajo y su sexualidad (Mies, 2019), enfatizando el carácter político inscrito, si no dicho, de las ciencias económicas. Por lo que nos interesa en este artículo, retomamos la cuestión de cómo la subordinación de las mujeres viene siendo producida de una manera muy poderosa, en la forma en que conceptualizamos el trabajo y, en particular, el trabajo de las mujeres.

Silvia Federici, en su obra seminal Calibán y la bruja (2010), analiza en detalle una parte de esta genealogía que se encuentra entre el colapso de la economía feudal europea y la aparición del nuevo orden capitalista que se consolidó con la expansión colonial de Europa. La autora explica que este cambio paradigmático solo fue posible porque se consiguió separar los medios de producción de los/as trabajadores/ as, transformar los cuerpos en máquinas de trabajo y someter a las mujeres a una función de reproducción de la fuerza laboral (2010: 85 y ss). Este nuevo orden, y la acumulación de capital que suscitó, llevó a procesos muy violentos que acarrearon la destrucción de los poderes de las mujeres, el exterminio de muchas de ellas tanto en Europa como en el extranjero, y la reinvención de una jerarquía basada en el género, la raza y la edad que se convirtieron en constituyentes de la dominación de clase (2010: 90). Argumenta que el capitalismo no fue una evolución dentro del sistema sino la contrarrevolución llevada a cabo por señores feudales, comerciantes ricos, obispos y papas para someter y destruir las luchas seculares por la emancipación de los/as pequeños/as agricultores/as, artesanos/as y trabajadores/as de las otras ordenaciones centrales de la economía política de la época (2010: 33-34). Este punto es capital para nuestro análisis porque arroja luz sobre dos cuestiones clave. La primera es Europa misma y su historia, mucho más plural de lo que ciertas opiniones homogeneizadoras y totalitarias del continente nos querrían hacer creer. La segunda es que en los intersticios de Europa hubo y hay formas de vida resistentes a este nuevo orden capitalista que perduran de muchas maneras en las sociabilidades. Como nos advierten las epistemologías del Sur, muchas sociabilidades y prácticas han quedado 
fuera del capitalismo, o fueron pensadas y llevadas a cabo en resistencia contra él. La cooperativa "Capuchinhas", como veremos a continuación, puede ser, en gran medida, un buen ejemplo de esto. De forma subalterna, clandestina o invisible, esta pluriversidad de proyectos de emancipación y visión de Europa y el mundo permanecen, si bien transformados. Están activos aunque en la superficie la realidad parezca totalmente dominada por la hegemonía del capitalismo contemporáneo.

Es en este contexto que surge la naturalización del "trabajo por amor" (Federici, 2013: 36). En otras palabras, la idea nace de la mujer como "ama de casa," cuya función es llevar a cabo, sin reconocimiento ni remuneración, todas las actividades que permiten que su descendencia se mantenga viva y pueda trabajar en fábricas, campos y negocios capitalistas. Así, se establece una de las formas que sustenta la acumulación de capital y la producción de riqueza: la explotación máxima del trabajo, ya sea asalariado, forzado o doméstico. El trabajo reproductivo de las mujeres y su subordinación se inventan y reifican a través de las ideas esencialistas de lo femenino como, naturalmente, cuidadoras y pertenecientes al espacio privado donde se inserta lo doméstico y que no puede alcanzar lo público. En otras palabras, la realidad está segregada y jerarquizada entre economía y vida, entre trabajo privado y público, productivo y reproductivo, y entre mujeres y hombres.

Sin embargo, si el trabajo se entendió durante mucho tiempo como "el otro nombre para actividades humanas, incluidas aquellas que no se realizan para la venta, sino por razones completamente diferentes, y que no pueden separarse del resto de la vida o ser almacenado"10 (Polanyi, 1975 [1944]: 72), con la Revolución Industrial moderna su mercantilización se profundiza. De ello se deduce que hay toda una esfera de actividad humana, a saber, el trabajo de las mujeres, que se lleva a cabo fuera del ámbito industrial y comercial, es decir, en el espacio doméstico o comunitario, que no tiene valor de cambio en el mercado, deja de ser trabajo, o como mucho se consideran como tales trabajos reproductivos. En palabras de Federici (2010: 111-112):

[Las] relaciones monetarias comenzaron a dominar la vida económica, [ellas] encontraron mayores dificultades que los hombres para mantenerse, así, se las confinó al trabajo reproductivo en el preciso momento en que este trabajo se estaba viendo absolutamente devaluado.

Al transformar el trabajo en una mercancía, aunque sea una mercancía ficticia ${ }^{11}$, como nos advierte Polanyi (1957 [1944]: 72), se ocultaron en la oscuridad impuesta a la esfera de lo privado, una enorme multiplicidad de prácticas, conocimientos, tecnologías, sabidurías, actividades, que aseguran la vida de muchas maneras. No es solo el trabajo implicado en la necesidad infinita de todas las tareas que garantizan la higiene, la alimentación, el refugio, la ropa, es decir, las condiciones fundamentales

10 Traducción de las autoras.

11 Al no ser el tema de este texto, no debemos dejar de señalar que la financiarización de la economía global contemporánea ha estado promoviendo cambios sustanciales en el concepto de trabajo, el acceso al trabajo y el derecho al trabajo. A medida que desmembramos cada vez más la articulación entre el trabajo y la producción de riqueza, parece que estamos en un momento de otra transición paradigmática en la que el trabajo para muchas personas en el mundo no es una mercancía ficticia sino una ficción irrealizable. 
para la producción prolongada de la vida ${ }^{12}$. También es el trabajo de la agricultura familiar o la pesca; trabajo comunitario y de ayuda mutua; uso comunitario de tierra y agua ${ }^{13}$, hornos, lavaderos o molinos; circuitos de intercambio recíproco en la comunidad o vecindario; circuitos comerciales de proximidad basados en excedentes locales; las pedagogías que sirven para educar preservan la identidad y la memoria social, la espiritualidad o el lenguaje; estrategias cara a cara y piel a piel para la resolución de conflictos; la literatura oral; la artesanía y el arte llamado popular. Son todas estas obras de creación y mantenimiento permanente de las condiciones inmanentes y trascendentes de la vida, las que son desarticuladas de la producción, del reconocimiento como actividades humanas fundamentales para la economía. Es sobre la base de esta destitución de la dignidad de la relación entre economía y vida, atribuida a las tareas y responsabilidades que, en un gran número de la sociedad, están garantizadas por mujeres de todas las edades, que se inventó lo que Amaia Orozco llama una "ética reaccionaria del cuidado" (2014: 148). Con este paso, no solo se subvirtió por completo la profunda imbricación entre la economía y la vida, sino que se crearon las condiciones para que hombres y mujeres naturalizaran las jerarquías y las desigualdades que resultan de ellas.

A pesar de su diversidad, la crítica feminista contemporánea ha sido muy elocuente al desarmar tres ideas primordiales que subyacen a la invención del trabajo reproductivo no remunerado de las mujeres. La primera es la construcción social del trabajo doméstico como trabajo de las mujeres y la satisfacción personal que debería derivarse de él (Federici, 2013: 5). Ellas han demostrado durante mucho tiempo que esta naturalización es una falacia y, por tanto, puede destruirse y superarse (Saffioti 1978; Gibson-Graham 1996; Federici 2013). Por otro lado, han estado revelando el valor económico, en términos cuantitativos, que estos trabajos tienen en el cálculo inclusivo de los presupuestos nacionales y el Producto Interior Bruto (Gottfried, 2013; Orozco, 2014; Jubeto, 2017). Finalmente, la crítica feminista ha estado demostrando la artificialidad de la separación de lo público y lo privado y de la economía y la vida (Gibson-Graham, 2008; Cunha, 2014, 2015, 2017). Reconociendo la construcción social de lo que consideran en muchos casos el trabajo de las mujeres, muestran de muchas maneras que lo privado también es político (Hanisch 1970; Patemen 1983) $)^{14}$ y que la producción de vida debe estar en el centro de la economía (Comerciante, 1990; Puleo, 2013; Shiva, 2018).

Sin embargo, la dicotomía entre el trabajo productivo y reproductivo sigue activa y, en nuestra opinión, continúa trabajando hacia la desigualdad y la subordinación de las actividades que el ciclo colonial europeo secular, el surgimiento del capitalismo y la reconfiguración de los sistemas patriarcales que se derivan de estos cambios, atribuye y obliga a las mujeres a adoptar como propias. Las experiencias socioeconómicas llevadas a cabo por muchas mujeres del Sur, lugar de sufrimiento, resistencia y alternativas, no solo disputan la hegemonía totalitaria de las dicotomías, sino que también las subvierten. La relación de oposición jerárquica entre trabajo productivo o

12 Utilizando el concepto de producción expandida de la vida, estamos introduciendo en el debate una crítica feminista de la idea de José Luís Coraggio $(2011,2015)$ de la reproducción expandida de la vida.

13 En Campo Benfeito, estos regímenes continúan en la práctica y tienen un nombre: la ayuda mutua y el trabajo comunitario del agua se llama levadas y de la tierra, las vessadas.

14 El lema "lo personal es político" tiene su origen en una obra con el mismo título escrito por Carol Hanisch. Fue publicado en 1970 como parte de una colección de ensayos editada por Shulamith Firestone e Anne Koedt, Notes from the Second Year: Women's Liberation. 
reproductivo, trabajo productivo e improductivo, comercio formal o informal, emancipación u opresión, privado o público, doméstico o político, amor o ira, voz o silencio, encarcelamiento o huida, género femenino o género masculino, poder o sujeción, rural o urbano, tradicional o moderno, sufrimiento o felicidad, está permanentemente desarticulada por las ideas y experiencias de las mujeres y da como resultado un pensamiento socioeconómico subalterno enunciado en otros términos. Para muchas de ellas, cocinar, cultivar, comerciar, tejer, enseñar, curar, fabricar medicamentos o establecer metas para sus negocios son parte de su repertorio diario de acción. Ni los espacios físicos, ni los tiempos, ni las habilidades y conocimientos necesarios, y mucho menos la consideración de que cualquiera de estas actividades sean productivas, improductivas o reproductivas, son parte de su pensamiento y de su mundo. Argumentamos, por tanto, como ya lo hemos hecho en otros textos (Cunha, 2015, 2017), que las obras de las mujeres, aquellas históricamente atribuidas a ellas, son las más productivas de todas porque las consideramos las fundadoras y alimentadoras de la vida, la dignidad, la imaginación cotidiana del futuro. Por ello, la realización de la justicia social tiene que ser cognitiva y sexual. Es sobre la base de esta discusión teórica que una hermenéutica feminista de epistemologías del Sur debería contribuir al desmantelamiento y la superación de la dicotomía entre el trabajo productivo y reproductivo. En otras palabras, consideramos que todas las tareas que las mujeres hacen o eligen realizar en cualquier esfera de la vida son productivas.

En la segunda parte de este texto, confiaremos en la vida y el trabajo de la cooperativa "Capuchinhas" para desarrollar y fundamentar nuestro argumento. En coautoría con Engrácia Duarte, Ester Duarte, Isabel Rodrigues, Henriqueta Ribeiro, queremos presentar la cooperativa y discutir tres temas que nos parecen fundamentales. El primero es la continuidad vital entre lo privado y lo público. El segundo es que una economía sostenible (Paulson, 2016) solo es posible con otra economía del tiempo y del deseo. El tercero es que el crecimiento económico puede ser lo opuesto a la abundancia y la emancipación.

En esta sección del texto, nuestra propuesta de redacción es que debe acercarse a un diálogo. Por un lado, las ideas, los recuerdos se convierten en palabras y también en observaciones que son el resultado de muchas conversaciones e interacciones mutuas a lo largo del tiempo, que constituyen nuestra etnografía. Por otro lado, están las reflexiones y análisis con los que seguimos el tiempo y el proceso de esta historia donde encajan muchas vidas. Para esto, presentamos una sutil diferencia visual para poder realizar, aunque sea imperfectamente, la conversación que queremos que esté presente en este texto. De esta forma, queremos practicar la co-autoría y subvertir la lógica extractivista de la producción de conocimiento. Sin embargo, es un ejercicio difícil y siempre incompleto, necesario para la hermenéutica feminista de las epistemologías del Sur que aquí llevamos a cabo.

\section{La cooperativa "Capuchinhas": donde lo personal es político y lo político es personal}

\subsection{Transformar conservando: el contexto y los comienzos de la cooperativa}

La cooperativa "Capuchinhas" funciona en la vieja escuela -deshabilitada en 1981 por falta de niños- en el pueblo de Campo Benfeito. Es parte de un territorio montañoso con pueblos dispersos y escasamente poblados. Se compone de cuatro muje- 
res, todas residentes en el pueblo: Engrácia Duarte, Ester Duarte, Isabel Rodrigues, Henriqueta Ribeiro. La mayoría de sus habitantes son personas mayores con fuertes lazos culturales y sociales con la región. El pueblo de Campo Benfeito tiene hoy 51 habitantes de los/as cuales 14 son niños y jóvenes. Las "Capuchinhas" son madres de 5 de estas/os habitantes más jóvenes.

En la década de 1980, la situación de aislamiento y falta de servicios públicos y oportunidades de empleo en la región era drástica. El país había salido hacía poco menos de una década de una larga dictadura que, entre otras consecuencias, causó mucho empobrecimiento, analfabetismo generalizado, falta de servicios públicos y un desarrollo territorial muy desequilibrado. La revolución democrática de 1974 inauguró un nuevo período político, marcado por una imaginación social que abrió la puerta a múltiples formas de emancipación y democratización de la sociedad. Es importante referirse a este contexto para comprender en qué medida las iniciativas que precedieron a la cooperativa "Capuchinhas" son, en sí mismas, un movimiento con un fuerte componente de cambio y transformación del viejo estado de cosas, particularmente para las mujeres.

A principios de la década de 1980, se celebró una reunión comunitaria para la gente de la cordillera de Montemuro con el objetivo de identificar las necesidades del territorio para la promoción de iniciativas económicas locales. Además de las autoridades locales, los residentes y las organizaciones sociales de la región, una de las organizaciones involucradas en este proceso fue el Institute for Cultural Affairs $(\mathrm{ICA})^{15}$. Fue en este contexto que se pensó que era una respuesta apropiada emprender un curso de "Corte y Costura", que tuvo lugar en 1985. El lugar elegido fue la escuela del pueblo donde ya se habían llevado a cabo otras iniciativas de animación para el desarrollo local, tales como: talleres de ocupación de tiempo libre para niños/ as y jóvenes, exámenes médicos, una colección de saberes ancestrales que resultó en un Cancionero y el texto Campo Benfeito: recuerdos de una época. La formadora elegida para el curso de "Corte y Costura" era una residente de la aldea, Cidália da Conceição, que conocía el oficio y las técnicas asociadas al mismo.

Esta elección aclaró dos cosas importantes. Por un lado, se reconoció que había mucho conocimiento propio, que no se había olvidado; por el contrario, todavía estaban presentes y activos en la aldea. Por otro lado, era evidente que los procesos necesitaban una dinámica anclada en las expectativas y deseos de las poblaciones y no de políticas de arriba hacia abajo.

Ella fue quien entrenó y formó un grupo de más de una decena de mujeres que en ese momento tenían sus proyectos de vida en intermitencia. Su apego al lugar donde nacieron y se criaron aún no las había expulsado de su aldea en busca de una oportunidad laboral remunerada que la montaña parecía incapaz de ofrecerles. La reconciliación entre quedarse y lograr la independencia parecía ser muy difícil.

Queríamos ser diferentes de nuestras madres, que siempre dependían de sus maridos para obtener dinero y hacer lo que mejor creyeran con él. Queríamos ser

15 Organización local destinada a aprovechar los recursos endógenos como estrategia para promover el desarrollo local. Funcionó en Mesio, otra aldea cercana, hasta 1995, cuando cesó sus actividades en el territorio. ICA Portugal formó parte de ICA International, que se ha establecido como una comunidad global de organizaciones sin fines de lucro que tiene como objetivo fomentar el desarrollo humano y operar de manera descentralizada. Para obtener más información, visite www.ica-international / org 
independientes, capaces de gobernarnos a nosotras mismas, tomar decisiones con autonomía y libertad. Para nosotras fue muy importante romper con algunas cosas del pasado, pero sin tener que renunciar al pueblo de Campo Benfeito.

Es necesario tener en cuenta que, con la revolución democrática en el país, surgió también el discurso de la necesidad de su modernización. Esto significaba, en algunos casos, pensar y describir las formas de vida en esos pueblos como tradicionales y obsoletas. En otras palabras, estas mujeres sentían profundamente una contradicción: el valor de la tierra, el conocimiento y las formas de vida asociadas a ella, y la idea de que, en estas condiciones, no podría darles lo que querían. Es importante tener en cuenta que estas subjetividades pudieron leer su mundo, identificar los problemas que enfrentaron y comprender las oportunidades que necesitaban construir para comenzar su proceso de transformación mientras conservaban lo que no querían renunciar (Tzul Tzul, 2015). En otras palabras, lo que está en juego, en nuestra opinión, es que para ellas las dicotomías entre tradición y modernidad, transformación y conservación, lo privado y lo público resultaron en un conflicto permanente porque no solo eran extraños para ellas, sino que no respondían a la realidad presente o futura que querían para sí mismas.

\subsection{La imaginación polifónica de la emancipación de las mujeres}

Cuando comenzó el curso de "Corte y Costura", Henriqueta trabajó para el ICA en Mesio $^{16}$. Su trabajo fue instrumental en las actividades de la asociación con los grupos locales de artesanos. Realizó estudios de mercado y logística organizada para participar en ferias. Sin embargo, Esther, que había terminado sus estudios, apoyaba las tareas de su familia y la economía doméstica, tanto en casa como en el campo. Engrácia e Isabel todavía estaban estudiando y, por tanto, no asistieron a esta formación inicial. Pero su vida se cruzará años después con las "Capuchinhas".

En 1986, la Comisión para la Condición Femenina $(\mathrm{CCF})^{17}$ se dio cuenta del trabajo de ICA en Montemuro y promovió otro curso de capacitación para varios grupos de artesanas, incluidas algunas de las capacitadas en "Corte y Costura" en Campo Benfeito. Cidália, Henriqueta y Esther fueron a Oporto para participar en esta sesión de capacitación sobre el tema "Gestión Profesión Mujer", financiado por el Fondo Social Europeo (FSE). Esther era menor y tuvo que pedir un permiso especial a su padre y a su madre para viajar y asistir al curso de formación. Para poder convencer a la familia, particularmente al padre, necesitó la ayuda de Maria Braga da $\mathrm{Cruz}^{18}$ y una anciana del pueblo.

Esta formación en la ciudad de Oporto tenía como objetivo preparar a las mujeres para su proceso de independencia económica. Esto incluía, entre otras cosas, darles habilidades prácticas de gestión para que pudieran dar cuenta de los costos de producción y calcular el valor y el precio final de sus productos, informarles sobre los pasos y documentos necesarios para legalizar sus actividades y cómo acercarse y tratar con clientes potenciales. Además, era parte del proyecto de capacitación

16 Una de las parroquias del consejo de Castro Daire.

17 Actualmente este organismo gubernamental se llama Comisión para la Ciudadanía e Igualdad de Género. Se puede encontrar más información al respecto en: https://www.cig.gov.pt/.

18 Ana María Braga da Cruz fue dirigente de CCF y luego presidenta, delegada regional y asesora principal de la Comisión para la Igualdad y Derechos de las Mujeres (CIDH) que vino después. 
para abordar un tema muy particular y desafiante para la época, que se llamaba "Ser Persona y Ser Mujer". Se pretendía trabajar con las subjetividades de estas mujeres, siempre teniendo en cuenta y respetando su propia forma de ver el mundo y la vida.

Esto surgió de muchas maneras y una de las cosas que más sorprendió a Maria Braga da Cruz fue el hecho de que no entendían por qué las tareas de aprendizaje y enseñanza estaban separadas de las tareas que sustentaban la vida del grupo, y por qué los trabajos considerados domésticos deberían considerarse un obstáculo a su emancipación.

Ellas en los intervalos entre las sesiones de entrenamiento, entraban a la cafetería para apoyar la preparación de las comidas. En nuestra opinión, a pesar de la apertura que queríamos mantener a estas subjetividades femeninas, tuvimos que hacerles entender que allí cocinar no era su responsabilidad. La única forma de lograr esto era involucrarlos aún más en temas de capacitación como la planificación familiar.

Que lo personal también es político, está muy bien tematizado en esta memoria y en esta descripción. Por un lado, vemos que llevaron a esta formación en Oporto lo que muchas feministas en ese momento aún no podían entender: la falacia de la separación entre lo público y lo privado, la formación y la vida, el arte y la artesanía. Mostraron en su forma simple de organizar el tiempo y las cosas que, al final, todos los trabajos de las mujeres eran productivos porque eran necesarios y contribuían al buen vivir de todo el grupo. Una vez más, su visión de sus vidas y prácticas puso en tela de juicio la racionalidad de la separación y la categorización que no captura ni las complejidades ni las articulaciones que forman parte de la vida. Por otro lado, contradecían con sus acciones la forma jerárquica en que se piensan las pedagogías y la formación: hay cuerpos de aprendizaje, cuerpos de enseñanza y otros cuerpos que están allí, pero que deben permanecer lo más invisibles posible. Y entre estos cuerpos, hay líneas abisales (Santos, 2018) que deben mantenerse.

El grado de subversión de su iniciativa de dejar el espacio de capacitación para ir a la cocina y colaborar en las comidas fue una gran molestia para las feministas de $\mathrm{CCF}$, quienes estaban convencidas de estar rescatándolas de su opresión, ofreciéndoles la comodidad de no realizar ninguna tarea considerada subordinada, como cocinar. Además, sentían la necesidad de abordar cuestiones que consideraban realmente relevantes para su liberación definitiva de lo que pensaban que era oscurantismo rural: la planificación familiar. Todo este episodio es una pieza de gran valor heurístico cuando deseamos hacer una hermenéutica feminista de las epistemologías del Sur. Destaca la reproducción del sometimiento de muchas mujeres y sus funciones sociales y cómo esto ha estado alimentando las desigualdades estructurales entre ellas. Además, a nuestro entender, sacan a la luz cómo los restos coloniales están inscritos en las racionalidades, incluso las feministas.

Fue durante esta formación que Adelaide Pereira, otra mujer de Montemuro que también las había acompañado en la primera sesión de formación en "Corte y Costura”, creó la imagen de la Capucha que todavía usa la Cooperativa.

\subsubsection{Objetos que son más que objetos y otra economía del deseo}

Es muy interesante que, también a este respecto, estas mujeres mostraran su capacidad para mantener su conexión con la tierra y los objetos que eran más que objetos 
porque significaban su pertenencia y su cultura, y, al mismo tiempo, proyectaban su imaginación del futuro. La capucha, esta capa de burel que les protegía del frío en invierno, se transformó en las "Capuchinhas", que, al no ser la misma cosa, se reflejan mutuamente y están unidas por esta lógica del matrimonio que, en lugar de separarse y ser desiguales, crea alianzas y convergencias. Argumentamos que esta economía del deseo, que reúne más que desarticula, es una de las bases de una economía porque no se nutre del desperdicio. Por el contrario, se alimenta de inventos y nuevos ciclos de vida que dan paso a ideas, cosas y todo tipo de recursos, tanto materiales como simbólicos.

\subsubsection{Quiero todo y quiero ser yo quien diga qué es todo para mí}

Después de estas actividades de preparación, el proyecto preveía el apoyo de una estilista para acompañar a cada uno de los grupos de artesanas que, mientras tanto, se estaban formando. Helena Cardoso, con una tradición familiar en la industria textil, fue seleccionada para trabajar con estas mujeres. Rápidamente se dio cuenta de que, a pesar de los esfuerzos realizados por el CCF, su formación en Oporto estaba muy lejos de la vida real y concreta de estas campesinas y sus aspiraciones, y que ni siquiera habían percibido, como decían, una emancipación para ellas. Todo esto se hizo evidente pronto, en los primeros días de contacto, cuando le pidió a cada una que seleccionara una foto con la que se identificara más estrechamente. Helena sintió en ese momento cómo sus prejuicios sobre estas mujeres eran poderosos, porque se sorprendió por la elección de Henriqueta: "Nunca olvidaré que Henriqueta eligió una foto del 25 de abril que tenía en su centro la frase: ¡Queremos todo!’. Hasta el día de hoy no ha olvidado esta elección y esta imagen, ni Henriqueta ha renunciado a esta demanda. Con ella continúa explicando por qué sigue siendo parte de la cooperativa "Capuchinhas": "Quiero todo y quiero ser yo quien diga qué es todo para mí".

Sin embargo, el IM-Swedish Development Partner ${ }^{19}$, una institución dedicada a combatir la pobreza y la exclusión social, había realizado un estudio que concluyó que la cordillera de Montemuro, en Portugal, era una de las áreas más vulnerables del país. Fue el contacto de esta organización con el $\mathrm{CCF}^{20}$ lo que creó la oportunidad de comenzar a trabajar estrechamente con cada uno de los grupos que habían pasado por este proceso de formación. Helena Cardoso comenzó apoyando al grupo "Lucha contra el Frío" en Relva ${ }^{21}$, seguido de las "Capuchinhas" en Campo Benfeito y, más tarde, las "Lançadeiras" de Picão ${ }^{22}$. Durante aproximadamente 8 años, se apoyó el trabajo creativo de producción, y cada grupo se benefició del apoyo financiero y el apoyo para mantener las condiciones materiales para la producción de ropa y accesorios hechos con recursos locales. Hasta el día de hoy, las "Capuchinhas" trabajan a partir de recursos endógenos que incluyen fibras de lana y lino, tintes naturales hechos de varios árboles de la región, a saber, roble y nogal, y se basan en los abundantes conocimientos y técnicas presentes en su comunidad. En 1987 se vendieron las primeras piezas, producidas por las manos de Cidália, Henriqueta y Esther. A partir de sábanas de lino en

\footnotetext{
19 Para obtener más información sobre esta organización, consúltese: https://www.imsweden.org/

20 Aunque no es el tema de nuestro texto, queremos enfatizar la fuerte articulación entre varias organizaciones sociales y organismos públicos que se estaba desarrollando en el terreno y que muestra que hubo una fuerte movilización para llevar a cabo lo que se pensaba que era la promoción de desarrollo local.

${ }_{21}$ Aldea de Rela, concejo de Castro Daire, distrito de Viseu.

22 Unión parroquial Picão y Ermida, municipio de Castro Daire, distrito de Viseu.
} 
desuso, se crearon las camisas. De las mantas de trapo, se hicieron chaquetas, y es en ese momento que también emergieron las primeras piezas en burel.

\subsection{Recursos locales, conocimientos y tecnologías ancestrales e innovación para una economía de abundancia}

Aquí es donde las "Capuchinhas" comienzan a reinventar el uso y la reutilización de los recursos disponibles. Inicialmente parece que esta estrategia tuvo más que ver con la falta de medios financieros para comprar telas $\mathrm{u}$ otras materias primas para trabajar. Pero, como se verá a continuación, esta perspectiva ha cambiado. Transformaron su forma de ver y valorar lo que, de hecho, constituían riquezas y a lo que nadie parecía dar la debida importancia. Esto incluye: tierra y productos de pastoreo como lino y lana; conocimiento de las propiedades químicas de las plantas, la corteza de los árboles, las flores y las raíces; tecnologías relacionadas con la producción de alimentos, tanto agrícolas como mecánicos, es decir, todo lo que formaba parte de la identidad y la colección cultural que se transmitía de generación en generación. Llegaron a integrar los principios de no desperdicio en sus creaciones al extender y renovar la utilidad material y social de muchas cosas. La sostenibilidad de los procesos de producción estaba en marcha, tanto por las viejas prácticas de reutilización como por las prácticas de reinvención e innovación que, en la cooperativa, comenzaban a consolidarse. Cidália era la que más experiencia poseía y la que tuvo mayor destreza en la fabricación de piezas, y esto se reflejó en el rendimiento que lograron con estas prendas. "En una feria, Cidália logró ganar 80 contos [equivalente a 400 euros] y solo logramos hacer 5 contos [equivalente a 25 euros] cada una".

\subsubsection{Obligando al mercado a estar al servicio de la transformación de la vida}

Esta observación merece una reflexión. Desde su creación, la cooperativa de las "Capuchinhas" siempre ha tenido la intención de generar ingresos financieros para que estas mujeres puedan cumplir su objetivo de ser independientes de dos maneras: rompiendo con la costumbre de que fueran los padres y esposos quienes administraran y decidieran qué hacer con el dinero de la familia; teniendo los medios materiales para decidir quedarse a vivir en su tierra. Este logro que opera tanto en un cambio de subjetividades como en la materialidad de la vida, fue y es primordial para ellas. Sin embargo, como se puede ver a lo largo del texto, no han sometido a la mercantilización por completo su vida o su trabajo. Su adhesión al mercado no está sujeta a sus condiciones, en el sentido liberal del término. Se niegan a convertirse en instrumentos del mercado: por el contrario, es el mercado el que se convierte en un instrumento de liberación para ellas. En este sentido, han desarrollado una relación estratégica con el mercado, que se ve moderada por sus elecciones, que incluyen mantener su estilo de vida y producción y su independencia personal.

Un año después, otra joven, Helena Félix, se unió al grupo "Capuchinhas", que permaneció cooperativo durante 16 años hasta que se mudó a la ciudad de Viseu por razones familiares. Quiso seguir estudiando en la universidad, pero no fue posible y abandonó los estudios. Hasta que se unió al grupo permaneció en su pueblo, Rossão, que está a $1 \mathrm{~km}$ de Campo Benfeito. Se fue metiendo en cursos de formación con la expectativa de utilizar algún día lo aprendido para obtener un trabajo remunerado que le permitiera ser "más autónoma, más independiente, más libre". Hasta ahora 
sabía que, para tener dinero, había de contar con el consentimiento y el acuerdo de su padre, a pesar de participar en todas las tareas agrícolas y domésticas. Trabajar en la cooperativa "Capuchinhas" fue, para Helena, el ejercicio de una profesión artesanal, tener su propio dinero y tener la capacidad de decidir sobre su uso. "Estar en las "Capuchinhas" fue aprender muchas cosas, como investigar el conocimiento ancestral de nuestros pueblos, valorar mi propio trabajo y también cómo cuidar a los clientes y la venta de las piezas hechas por mí y mis compañeras".

Los siguientes pasos fueron el registro de la marca "Capuchinhas", con la que se nombraban desde el principio, la profesionalización que requería mejoras en algunas técnicas, y la introducción de técnicas de tejeduría. Reclutaron a las mujeres mayores de la aldea que sabían tejer y manejar la urdimbre de los telares. Una de estas entrenadoras locales era María Augusta Carneiro, ahora de 91 años y la persona más vieja de la aldea, que hacía mucho tiempo hacía colchas de trapos ${ }^{23}$, sábanas, alfombras y cubiertas de pelusa ${ }^{24}$. Ella afirma, aun hoy, que en trabajos como el de los telares "el tiempo no se paga". Explica que esto significa que el tiempo no tiene precio y que lo que se hace no tiene la mera función de ser una mercancía con la que se busca acumular capital. María Augusta hace explícita su convicción cuando ilustra su pensamiento con el episodio de la colcha y el pañuelo.

Quería un nuevo pañuelo para los días de fiesta e ir a misa. Fue entonces cuando decidí hacer una colcha para vender. Fui a Lamego a la feria y vi cuánto costaba el pañuelo ${ }^{25}$. Decidí vender la colcha por el valor del pañuelo porque eso era lo que quería. Gané 100 escudos y gasté 100 escudos $^{26}$. Todavía tengo el pañuelo hoy y lo uso con frecuencia.

\subsubsection{El tiempo no es dinero ni el dinero puede ser tiempo}

Hemos estado argumentando que la sostenibilidad, en sus diferentes facetas, tiene que incorporar otra economía del tiempo y del deseo. Primero, porque en los procesos productivos de la vida no se puede pensar en el tiempo de la misma manera que en la fabricación industrial o en la era digital. La repetición necesaria para estas actividades humanas es una sintonía constante entre las necesidades y lo que tenemos que satisfacer para ellas, y eso requiere tiempo y creatividad. No se puede separar una cosa de la otra. Ahora bien, para que eso suceda el tiempo tiene que ser entendido de una manera no instrumental y no lineal. El tiempo produce tiempo, ya que la vida produce vida y el tiempo no puede calcularse ni convertirse en un precio sin restringir su valor sustantivo en la producción de vida. Pero esta economía del tiempo implica otra economía del deseo en la que la moderación y la sobriedad son elementos clave. Lo que se desea debe estar en equilibrio con el tiempo requerido para esta producción de vida. El tiempo no es dinero ni el dinero puede ser tiempo. El vértigo de la acumulación, que es la economía del deseo de nuestro presente capitalista, oculta que para que algunos acumulen,

23 Técnica de reutilización de tejidos, creando piezas para apoyar el cultivo, es decir, para extender y secar las semillas.

24 Se refiere a una técnica local de "tirar" de los hilos en la tela, que dan lugar a patrones específicos en los tejidos finales. No todas las mujeres sabían cómo hacer esta técnica porque requería una mayor experiencia y conocimiento en el arte de tejer.

25 Producido industrialmente.

26 Equivalente a $0,50 €$. 
muchos deben ser despojados. Esto significa quitarles el tiempo de vivir, el tiempo de ser, el tiempo de pensar, de regocijarse, o de hacer sus duelos. El tiempo se convierte en un caníbal de sí mismo generando la reproducción masiva de las injusticias sociales. Así, la sobriedad del deseo no es su renuncia ni una defensa del empobrecimiento o la austeridad neoliberal. Más bien, es el reconocimiento de que la innovación no está en la obsolescencia programada y forzada de cosas, ideas y personas. La economía política de esta sobriedad convoca epistemologías del cuidado y del no desperdicio. Lo que María Augusta hizo al cambiar su edredón por su pañuelo con monedas es una declaración inequívoca de que lo que más importa no son los precios o el valor de mercado, sino el valor del uso de las cosas. Y este valor siempre es contextual y se construye en una relación en la que el tiempo y el deseo no pueden reducirse a meras mercancías desgajadas de su historicidad y de la matriz donde ocurre la vida (Cunha, 2015).

Su trabajo como formadora en la cooperativa comenzó con cierta resistencia por su parte. No sabía precisar si fue entre 1989 y 1990. Para María Augusta, adaptar la técnica de producir colchas y alfombras a la ropa era "ridículo. ¿Dónde se ha visto esto en la vida? ¿La felpa así? ¿Te gusta? ¡Esto es para las colchas! “. Pero fue por sus manos que salió del telar el primer abrigo tejido y diseñado por Helena Cardoso y que tuvo como clienta a Maria Braga da Cruz. A finales de la década de 1990, María Augusta decidió retirarse: se alejó de la cooperativa, desmanteló su telar y no volvió a tejer. Pero toda su sabiduría y técnicas fueron transmitidas a Engrácia Félix Duarte, quien hoy es la guardiana y tejedora de la cooperativa.

Para estas diferentes generaciones de mujeres tejer lana o lino tiene significados diferentes. Se han diseñado nuevas funciones prácticas para estos tejidos, así como una nueva estética. Sin embargo, el conocimiento que ha circulado entre generaciones y que preserva las relaciones con la tierra, con la cultura, con las tecnologías de producción, sigue presente en este arte de inventar y expandirse de lo que está disponible en el mundo. La repetición con innovación es lo que caracteriza este trabajo. En este punto podemos ver que no es una obra de reproducción en el sentido de duplicación infinita, y que lo doméstico y lo público están en continuidad. Lo que se ha aprendido en el hogar, para la vida en la casa, es relevante y es lo mismo que lo que se produce para fuera del hogar. En otras palabras, las competencias son movilizadas siempre que sea necesario y en los diversos espacios-tiempos de la vida.

Engracia todavía estaba estudiando cuando su vida se cruzó con la historia de las "Capuchinhas". En las vacaciones de invierno y en su tiempo libre ayudaba en la vieja escuela donde se habían instalado. Fue ella quien comenzó a hacer zapatos de burel que son una marca registrada de las "Capuchinhas". Era una mujer joven acostumbrada a participar en la vida colectiva de la aldea y las iniciativas que allí se desarrollaban. Tomó un curso de teatro con el ICA, participó en la recolección del cancionero e hizo una gira con varias obras por la región. En 1993, fue invitada a meterse en la cooperativa porque había una necesidad de una persona más joven que pudiera dominar las técnicas de tejido. Ya había aprendido muchas cosas de su madre, que también tejía, pero fue con María Augusta que aprendió su técnica y su apego al oficio y al arte de los telares. Rápidamente se dio cuenta al unirse a las "Capuchinhas" que tenía habilidades y recursos que le permitirían vivir en su pueblo y criar a su familia donde siempre soñó hacerlo.

Ofelia Cabral, la madre de Ester Duarte, también sabía tejer y estaba orgullosa de lo que su hija estaba ayudando a crear en el pueblo para las mujeres. En 1994 fue invitada a unirse al grupo para reforzarlo. "Por mi parte, ni siquiera lo pensé dos veces y acepté, pero tenía que hablar con mi esposo, por supuesto. Estuvo de acuerdo, 
siempre que no fuera a la cooperativa en el verano porque había mucho trabajo con el heno y me necesitaba".

Decidió mantener sus ingresos en casa. Sabía que, si lo ponía en el banco, en la cuenta conjunta, seguiría siendo su marido el que cuidaría el dinero y decidiría qué hacer con él. Pidió el pago en especie, y nunca con cheques, para mantener libres sus decisiones con respecto a su dinero. Se sentía feliz por poder decidir cómo gastarlo; lo usó principalmente para renovar y mejorar su hogar. Uno de sus mayores placeres era no tener que rendir cuentas o negociar con su esposo por el uso de cada céntimo. Esto todavía le hace sonreír y pensar en sí misma como una mujer diferente y libre. El trabajo de Ofelia en la cooperativa no la liberó inmediatamente de ninguno de los otros trabajos que eran de su responsabilidad. Se levantaba temprano para preparar el almuerzo y cuidar de los animales. Regresaba de la cooperativa y sabía que todavía tenía que enfrentarse al trabajo agrícola en la granja y a las tareas de la casa al final del día. Para el verano, sabía que la dureza de la recolección y el tratamiento del heno para vender aumentaba, y la presión para que dejase de ir a las "Capuchinhas" también. Ofelia sabía que había una máquina llamada ensamblador que podría liberarla de este trabajo para poder tejer con las compañeras de la cooperativa. "Mi esposo no quería saber de gastar dinero para comprar el ensamblador ${ }^{27}$, pero le pregunté a mi hijo cuánto costaba y dónde comprarlo. Mi esposo juzgó que no podía pagarlo, pero junté el dinero y la compré". Ofelia no tiene dudas cuando dice que "el tiempo más hermoso de mi vida fue cuando estuve en las Capuchinhas. ¡Es bueno ganar dinero nuestro! ¡El día se pasaba sin darse una cuenta!”. Un problema de salud le hizo alejarse de los telares y del trabajo en las "Capuchinhas", pero la semilla y la siembra del compañerismo, autonomía, libertad y el poder de decisión habían cambiado para siempre la forma en que se ve a sí misma y el mundo.

La última en unirse a la cooperativa fue Isabel Maria Duarte Rodrigues. Esto sucedió en 1996, aún no había alcanzado la mayoría de edad. Al igual que Esther, después de terminar la escuela cuando era adolescente, se unió a su familia en tareas domésticas y agrícolas. Al principio no estaba segura de si eso era lo que quería y aceptó pasar por la experiencia. Como era muy joven, las "Capuchinhas" se sintieron bien y estuvieron de acuerdo con la idea. A Isabel le gustó lo que aprendió y se convirtió en una de ellas. Quería vivir en Campo Benfeito y, al mismo tiempo, quería tener un salario que le permitiera tener autonomía y hacer con la vida lo que ella pensaba que era mejor. Hoy en día, dice que traspasó a su familia la manera de hacer las cosas de la cooperativa. "En casa hablamos y decidimos juntos, y cada uno tiene sus propias tareas para que todo salga bien. Hay muchas cosas de las que no tengo que preocuparme y mantengo mi independencia. No podría ser de otra manera. Pero mi marido es muy compañero".

\subsection{Las exigencias y limitaciones de las políticas europeas y la reinvención de su autonomía}

No fue sino hasta el año 2000 que las "Capuchinhas" formalizaron su cooperativa en términos legales y obtuvieron el apoyo financiero del programa ESF LEADER para la creación de dos puestos de trabajo asalariados. Esta decisión se tomó a la luz de las nuevas necesidades que surgieron: la necesidad de realizar mejoras en la escuela donde se habían instalado y el requisito de una constitución formal para

27 Es un artefacto mecánico que recolecta heno, lo que reduce en gran medida el trabajo manual requerido para esta operación. 
poder solicitar apoyo financiero y programas de formación promovidos por agencias gubernamentales. Por otro lado, su contable las ha alertado sobre algunos beneficios de tal estatus legal, en lo que se refiere a la exención del impuesto IRC ${ }^{28}$. Sin embargo, la creación de la cooperativa tuvo implicaciones directas en el cálculo del valor de mercado de sus productos. Ahora tenían que incluir el IVA ${ }^{29}$, que era el valor de sus ingresos, y tenían que comenzar a cobrar los costos de la mano de obra (horas de trabajo) relacionados con el pago a la Seguridad Social. Este tipo de aumento de costos se percibió como un obstáculo que había que superar y una injusticia porque "la aplicación del impuesto no hace distinción entre el origen de los productos ni el modo de producción”. Esto significa que es el modelo industrial el que predomina, lo que socava estas otras economías "como nuestra cooperativa".

La forma en que se calculan los costos de producción es la siguiente: la primera pieza de cada colección sirve como referencia para el resto del mismo modelo. Primero se contabiliza la cantidad de tela e hilo utilizada. Luego se registra el tiempo requerido para su ejecución más los costos de la Seguridad Social. Cada hora de trabajo está indexada al salario mínimo nacional. Se agrega el IVA y esto resulta en el valor comercial final de cada producto. Cuando se trata de piezas que usan trabajo de tejido, el cálculo tiene en cuenta que hay tareas que deben ser realizadas por más de una persona. Por lo tanto, cada vez se cuenta el tiempo de cada una teniendo esto un impacto en el precio final. Engracia es la única que hace mallas y comparte con Isabel el tejido. Henriqueta y Esther son responsables de la costura. Las piezas se hacen a pedido y cada una decide el ritmo y la cantidad de piezas que desean realizar teniendo en cuenta sus metas personales en términos de ingresos y los objetivos de la cooperativa en términos de oferta a las y los clientes. Además, hay más tareas a realizar en la cooperativa que se comparten entre todas. Cada día de la semana cada una es responsable de realizar tareas administrativas, como servicio de correo, lectura de correos electrónicos, procesamiento de pedidos, soporte contable y servicio al cliente. Los viernes son los días dedicados a ayudarse mutuamente a hacer todo lo que queda y que no se ha atribuido a nadie en particular. La participación en ferias u otros eventos pasa por el mismo proceso de contabilización de las horas de trabajo y todo se registra en un cuaderno individual. Cada una controla su tiempo y trabajo, y la base de este modelo de gestión es la confianza interpersonal: "no necesitamos revisar los cuadernos de las demás".

La cooperativa de las "Capuchinhas" es entendida por nosotras como una economía que no apunta a la acumulación de capital y en la cual el control de los modos y tiempos de producción es decidido por el grupo. Hay varias características que podemos identificar en esta socio-economía cooperativa que nos llevan a afirmar que estamos ante una economía en la que la vida y la emancipación están en el centro, y donde la colaboración es el principal significado.

La primera es que sus productos no se venden en consignación porque eso significaría renunciar al control de parte del circuito de producción-comercialización y transferir decisiones sobre su trabajo, recursos y tiempo dedicado, a otros. Prefieren que se considere toda la cadena de producción como un todo sobre el cual deciden de manera autónoma. La segunda es la relación que establecen con las diseñadoras que trabajan con ellas. Saben que necesitan movilizar tales habilidades fuera de su grupo,

Impuesto sobre el Rendimiento de Personas Colectivas. En España sobre la renta de las empresas.

Impuesto sobre Valor Acrescentado. 
pero no se comprometen en el manejo de esta relación. Como dice la diseñadora actual de la cooperativa, Paula Caria, son dueñas de su proyecto. Eso es lo que las puso en sintonía. No existe una propiedad individual de la producción y se niegan a renunciar a tener la última palabra sobre lo que producen. El diseño de las colecciones se ha pagado como un proveedor de servicios, dándoles libertad para cambiar según las necesidades de sus clientes. Esto significa que pueden personalizar las piezas finales y alejarse deliberadamente de cualquier modelo de industrialización. Esto se hace aún más claro por su resistencia a adherirse a otros modos de producción o a iniciativas que, siguiendo el mercado como medida de todas las cosas, los proyectarían a un nivel comercial más competitivo y, por lo tanto, más rentable en términos de acumulación de capital. Incluso hay un conflicto discursivo entre las diseñadoras que trabajan para ellas y las mujeres de las "Capuchinhas". Las primeras sostienen que su proyección en otros mercados se correspondería con una mayor valorización económica de las piezas producidas $\mathrm{y}$, por lo tanto, más dinero para todas las personas involucradas. Desde la perspectiva de las segundas, la cooperativa es su espacio para la autonomía financiera, pero no puede convertirse en una nueva sujeción, en este caso al mercado. Consciente de esto, su cooperativa es una economía de crecimiento controlado. Mejor aún, esta opción indica que distinguen entre crecimiento económico y bienestar, buen vivir, felicidad y libertad. Producen lo que tienen la capacidad de producir y trabajan colectivamente para garantizar su salario y la existencia de su unidad de producción.

Con esta forma de conducir y administrar sus actividades, afirman que hay vida más allá de su cooperativa. Por lo tanto, manejan sus horarios y su dedicación a las "Capuchinhas" de acuerdo con lo que quieren seguir haciendo en la comunidad y la familia. Para ellas, su emancipación implica renunciar a la esclavitud por dinero, como dicen. Su trabajo debe significar vivir bien y con abundancia de lo que les es esencial: dignidad, respeto y tiempo para disfrutar todo lo que puedan por sí mismas, sus familias y sus comunidades.

Cada una trabaja hacia una meta salarial: poder producir para alcanzar el Salario Mínimo Nacional (SMN) a fin de mes. Isabel sabe que sus "hermanos ganan más en la ciudad, pero está segura de que en Campo Benfeito vive más feliz". Ganan más, pero también gastan más, no solo porque la vida en la ciudad es más cara, "sino también porque las demandas de consumo son mayores". Calculan que con el salario mínimo en la aldea no solo pueden satisfacer las necesidades familiares sino que también pueden ahorrar. Además, es posible disponer de los horarios de acuerdo con las necesidades de sus hijas e hijos trabajando en función de los diferentes momentos del día o del año. Sin embargo, cualquier persona que quiera ganar más de un salario mínimo puede hacerlo, ya que todas las horas de trabajo se cuentan y se pagan al final del mes.

Es interesante notar que, desde el principio, las especialistas en emancipación de mujeres, como María Braga da Cruz, consideraron que sus precios no calculaban todos los costos de producción. También se argumentó que estas mujeres no sabían cómo valorar adecuadamente sus conocimientos porque, en su mentalidad tradicional, la producción de ropa de abrigo o accesorios para el hogar eran fruto de los tiempos de miseria y de las tareas que las mujeres tenían encomendadas sin reconocimiento ni recompensa. Sin embargo, si observamos cómo hacen sus cálculos para definir el valor comercial de cada pieza, podemos ver que esta narrativa feminista es potencialmente ciega a otras racionalidades feministas que reclaman por sí mis- 
mas el poder de decidir su vida. Sostenemos que la emancipación de las mujeres es siempre contextual y, por lo tanto, entendemos que el modelo de gestión y fijación de precios adoptado por ellas, en este caso, responde a lo que parece más pertinente y más relevante para su forma de sentirse dueñas de su cooperativa y de sus vidas.

Las "Capuchinhas" siempre se consideraron como una cooperativa y no fue la formalización lo que las constituyó como tales. Están vinculadas por la confianza mutua, la reciprocidad y la colaboración, y han hecho de esto la base principal para su forma de producir, vivir y relacionarse con su entorno y comunidad. La cooperativa "Capuchinhas" es una historia de vida en la que caben muchas vidas. El lenguaje de la igualdad de género todavía les hace sonreír cuando se trata del impacto de su proyecto y la economía que han generado para ellas y para la región. Muy poco preocupadas por el concepto, continúan dando su propio contenido a su forma de ser iguales y diferentes de los hombres con los que tratan en sus vidas. Saben que, a su manera, la cooperativa "Capuchinhas" representa, para muchas mujeres en la Sierra do Montemuro, y más allá, una cara concreta de la emancipación sin tener que renunciar a la tierra, a la casa o la forma de ser felices.

\section{Notas Finales}

En un momento en que el análisis social está lleno de conceptos marcados por prefijos como postcolonial, post-social, post-confianza, post-político, entre otros, la noción de fracaso del conocimiento, o impotencia, y epistemologías centradas en ella, se hace evidente en la herencia moderna occidental. La hermenéutica feminista de las epistemologías del Sur extiende la atención necesaria a las resistencias y alternativas más invisibles porque están secularmente subordinadas. Entendemos, por lo tanto, que esta propuesta teórica que llevamos a cabo aquí va en contra del pesimismo sin salida del posmodernismo occidental y eleva la esperanza a una categoría epistemológica. Nos sentimos herederas de las muchas intelectuales feministas que han criticado durante mucho tiempo la idea de que el conocimiento científico es un conocimiento abstracto que no llega a ningún lugar ni tiempo. En cambio, ellas y nosotras proponemos que todo el conocimiento, incluido el conocimiento científico, es situado y debe ser humilde (Haraway, 1988; 1997). Es en este sentido que nos hemos propuesto colocar en evidencia la historia de la cooperativa de las "Capuchinhas" y todo lo que han logrado hasta la fecha. No escondemos las dificultades o contradicciones inherentes a una realidad que siempre es compleja, pero tomamos una decisión teórica y empírica para traer al debate lo que finalmente resiste y se configura como una alternativa concreta, viable y sostenible.

Somos conscientes de que quedan muchos problemas por abordar, pero lo mismo ocurre con todos los textos: son siempre y solo una parte mínima del conocimiento disponible. Aun así, en términos teóricos, discutimos varios temas, como la persistencia del sometimiento de las mujeres y la ampliación del concepto de economía. Lanzamos el desafío de pensar que la dicotomía entre el trabajo productivo y reproductivo esconde más de lo que muestra sobre las alternativas económicas pensadas y dirigidas por las mujeres. Además, este desafío sirve para pensar que, al mantener la dicotomía, no nos liberamos de la jerarquía que establece. Esto significa, desde nuestro punto de vista, que debemos comenzar a pensar en los trabajos de las mujeres, incluso en aquellos que vinieron con la opresión capitalista, colonial y patriarcal, 
como trabajo productivo. Solo de esta manera podremos hacer no solo visible sino también central un conjunto completo de actividades humanas que son los fundamentos primordiales de la vida para todos.

Desde el trabajo etnográfico colaborativo y en coautoría con las "Capuchinhas" hemos presentado, aunque brevemente, el contexto y la complejidad del surgimiento de este grupo de mujeres. Trajimos sus vidas, sus ideas, sus palabras, sus análisis de su mundo, y cómo quieren vivir libres y felices en él. Cómo quieren responder a la mercantilización progresiva de la vida y qué opciones les ha supuesto. Ellas y nosotras buscamos comprender cómo sus prácticas sustentan y se sustentan en la vida biológica y cultural y en la colección ancestral de conocimientos y tecnologías presentes en su cultura y territorio. En este texto argumentamos que el lugar del conocimiento y el trabajo de las mujeres en la resistencia y en la creación de alternativas concretas y viables siempre es productivo. En estas condiciones, sostenemos que la economía puede transformarse en relaciones sociales más solidarias, justas y sostenibles, es decir, para producir, proporcionar y redistribuir la vida en abundancia en todas sus formas.

\section{Bibliografía}

Amadiume, Ifi (1998). Male daughters, female husbands: gender and sex in African society. London: New York: Zed Books.

Armendariz Echániz, Eba (Coord.) (2018), Economia Feminista. Visibilizar lo Invisible. Dossieres EsF, $n^{\circ} 29$, Primavera, Economistas sin Fronteras.

Bidaseca, Karina; Vazquez Laba, Vanesa (Comps) (2011). Feminismos y poscolonialidad Descolonizando el feminismo desde y en América Latina. Buenos Aires: Ediciones Godot.

Butler, Judith (2004). Precarious life. The powers of mourning and violence. London, New York: Verso.

Carty, Linda; Talpade Mohanty, Chandra (2014). "Mapping Transnational Feminist Engagements: Neoliberalism and the Politics of Solidarity", en R. Baksh, W. Harcourt (Eds.), The Oxford Handbook of Transnational Feminist Movements. Oxford: Oxford University Press, 82-115.

Carrasco, Cristina (2003). "La sostenibilidad de la vida humana: ¿un asunto de mujeres?”, en M. León (Comp.). Mujeres y trabajo: cambios impostergables. Porto Alegre: Veraz Comunicação, 11-49.

Cunha, Teresa (2014). Never Trust Sindarela. Feminismos, Pós-colonialismos, Moçambique e Timor-Leste. Coimbra: Edições Almedina.

- (2015). Women inPower Women: outras economias geradas e lideradas por mulheres no Sul não-imperial. Buenos Aires: CLACSO-CODESRIA-IDEAs.

- (2017). "Todo o trabalho é produtivo: economias de abundância e da sobriedade", en X. Hermida González (Coord.), [Re]Pensar a democracia. Compostela: Foro obencomún, 101-134.

Federici, Silvia (2010). Calibán y la bruja: mujeres, cuerpo y acumulación originaria. Madrid: Traficantes de Sueños.

- (2013). Revolución en punto cero. Trabajo doméstico, reproducción y luchas feministas. Madrid: Traficantes de Sueños.

Gibson-Graham, Julie Katherine (1996). The end of capitalism (as we know it): a feminist critique of political economy. Oxford and Cambridge, MA: Blackwell Publishers. 
- (2008), "Diverse Economies: Performative Practices for 'Other Worlds". Progress in Human Geography, 32 (5): 613-632.

Gottfried, Heidi (2013). Gender, Work, and Economy: Unpacking the Global Economy. Cambridge, UK; Malden, MA, USA: Polity Press.

Hanisch, Carol (1970), "The personal is political" in Shulamith Firestone, Anne Koedt (Eds), Notes from the Second Year: Women's Liberation. Major Writings of the Radical Feminists. New York.

Haraway, Donna (1997). Modest_Witness@Second-Millennium.Female Man@ Meets OncoMouse ${ }^{T M}$ : Feminism and Technoscience. London: Routledge.

- (1988). "Situated knowledges: The science question in feminism and the privilege of partial perspective". Feminist Studies, 14(3): 575-599.

Jubeto, Yolanda (2017). "El análisis presupuestario con enfoque de género: un instrumento feminista clave para avanzar en la equidad socioeconomic". Ekonomiaz, 91: 300-329.

Lugones, María (2010). “Toward a Decolonial Feminism”. Hypatia, 25(4): 742-759.

Marçal, Katrine (2015). Who cooked Adam Smith's Dinner? A story about women and Economy. New York: Pegasus Book, Ltd.

Martins, Catarina (2016). "Nós e as Mulheres dos Outros. Feminismos entre o Norte e a África”, en A. Sousa Ribeiro, M. Calafate Ribeiro (Coords.), Geometrias da Memória: configurações pós-coloniais. Porto: Afrontamento, 251-277.

Merchant, Carolyn (1990 [1980]). The Death of Nature: Women, Ecology, and the Scientific Revolution. San Francisco: Harper \& Row Publishers.

Mies, Maria (2019). Patriarcado y Acumulación a Escala Mundial. Madrid: Traficantes de Sueños.

Osório, Daniela (2014). "Economía Solidaria e interdependência: Aportes desde perspectivas feministas", Quaderns de Psicologia. Vol. 16, № 1, 153-165.

Oyewùmí, Oyèrónkẹ (Eds.) (2005). African gender studies: a reader. New York: Palgrave Macmilliam.

Patemen, Carol (1983). "Feminist Critiques of the Public/Private Dichotomy", en S. I. Benn, G. F. Gaus (Eds.), Public and Private in Social Life. New York: St. Martin's Press, 281303.

Paulson, Susan (2016). “Ecologia Política”, en G. d'Alisa, F. Demaria, G. Kallis (Coords.), Decrescimento. Vocabulário para um mundo novo. Porto Alegre: TOMO Editorial, 63-67.

Pérez Orozco, Amaia (2014). Subversión feminista de la economía. Madrid: Traficantes de Sueños.

Pérez Orozco, Amaia; Agenjo Calderón, Astrid (2018). "Economía Feminista: Viva, Abierta y Subversiva", en E. Armendariz Echániz (Coord.), Economia Feminista. Visibilizar lo Invisible. Dossieres EsF n 29, Primavera, 6-10.

Polany, Karl (1957 [1944]). The great transformation. The political and economic origins of our time. Boston: Beacon Press.

Puleo, Alicia H. (2013). Ecofeminismo para otro mundo posible. Madrid: Cátedra. Colección Feminismos.

Rivera Cusicanqui, Silvia (2010). Ch'ixinakax utxiwa: una reflexión sobre prácticas y discursos descolonizadores. Buenos Aires: Tinta Limón.

Saffioti, Heleieth. (1978). Emprego doméstico e capitalismo, Petrópolis: Vozes.

Santos, Boaventura de Sousa (2014). The Epistemologies of the South, Justice against Epistemicide. Blouder - London: Paradigm Publishers.

- (2018). The End of the Cognitive Empire. The Coming of Age of Epistemologies of the South. Durham and London: Duke University Press. 
Shiva, Vandana (2018). ¿Quién alimenta realmente el mundo? El fracaso de la agricultura industrial y la promesa de la agroecología. Madrid: Capitán Swing.

Talpade Mohanty, Chandra (1991). "Under western eyes - Feminist scholarship and colonial discourses", en C. Talpade Mohanty et al. (Eds.), Third world women and the politics of feminism. Bloomington: Indiana University Press, 462-487.

Tzul Tzul, Gladys (2015). "Sistemas de gobierno comunal indígena: la organización de la reproducción de la vida", en L. Linsalata, H. Salaza (Coords.), El Apantle, revista de estudios comunitarios. Puebla: Sociedad Comunitaria de Estudios Estratégicos, 126-140.

Waterhouse, Rachel; Vifhuizen, Carin (Coords.) (2001). Estratégias das mulheres, proveito dos homens. Moçambique: Núcleo de Estudos da Terra. 